\title{
Introduction to Issue on Integrated Optics and Optoelectronics Part 1
}

B EING editors of this Special Issue, of the IEEE JOURNAL OF SELECTED TOPICS IN QUANTUM ELECTRONICS, we are excited, apologetic, thankful, and proud.

We are excited with the record number of submissions (95 in total), the technical advances contained in these papers, and the wide range of coverage of the subjects. The papers in this Special Issue document significant advances of the field at several fronts, which is an encouraging sign for the steady recovery of the telecom industry and the emergence of new opportunities.

We apologize to many authors for our slower responses than desired to get the reviewers' feedback to them and the relatively short time for them to make revisions. We also apologize for publishing some of the papers at a later date. Because of the space limit in each volume, we had to divide this issue into two consecutive volumes and publish the second volume as the March/April issue.

We owe special thanks to the more than 200 reviewers. Our reviewers are perhaps the busiest people in our profession. However, the responses from all of them have been magnificent. We appreciate their technical insight and willingness to share it with us. As editors, we are amazed how much the quality of our issue has been improved because of the thorough work of our reviewers.

We are proud that as a community (with more than 500 people contributing to and participating in the issue as authors or reviewers) we are making significant contributions to the technological foundations of optoelectronics. This issue documents

Digital Object Identifier 10.1109/JSTQE.2004.842340 advances in optoelectronic devices and circuits, both in theoretical and technological aspects. For people deeply involved in the field, you might find such improvements incremental. However, if you ever have a chance to read the IEEE JOURNAL OF SELECTED TOPICS IN QUANTUM ELECTRONICS on the same topic published more than two years ago, you will surely be amazed by the great accomplishments the society has made collectively over this relatively short period of time.

Last, but not least, we want to express our deepest thanks and appreciation to the JSTQE staff who have worked tirelessly with us to complete this issue. They are Janet Reed, Eileen Murray, and Linda Matarazzo. They have been more efficient than we have thought possible, and we cannot thank them enough. If you find any mistakes in or are not satisfied with this issue, the responsibility is ours. The JSTQE staff deserves nothing but our praise.

Yu-HwA Lo, Guest Editor

University of California San Diego

Department of Electrical and Computer Engineering La Jolla, CA 92093 USA

KUNISHIGE OE, Guest Editor

Kyoto Institute of Technology

Kyoto, 606-8585 Japan

RoEl BAETS, Guest Editor

University of Ghent-IMEC

Department of Information Technology (INTEC)

Ghent, B-9000 Belgium 


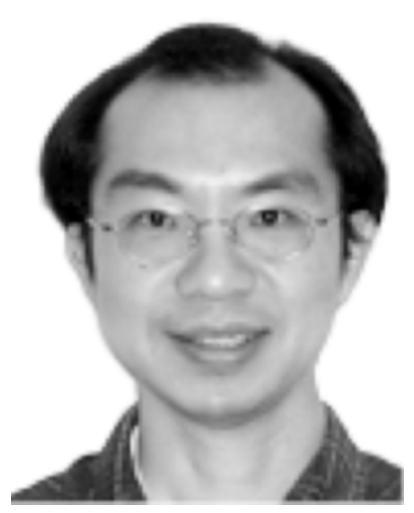

Yu-Hwa Lo received the B.S. degree from National Taiwan University, Taipei, Taiwan, R.O.C., in 1981, and the M.S. and Ph.D. degrees from the University of California, Berkeley, in 1986 and 1987, respectively, all in electrical engineering. His Ph.D. research was on molecular beam epitaxial growth and characterization of GaAs-on-Si heterostructure.

From 1988 to 1990, he was a Member of Technical Staff at Bellcore (now Telcordia), Piscataway, NJ. His research at Bellcore was focused on OEIC transmitters and WDM laser arrays for telecommunication. He joined the Faculty of School of Electrical Engineering, Cornell University, Ithaca, NY, in 1991, as an Assistant Professor, and then became an Associate Professor in 1996. His research group at Cornell did research on semiconductor materials and optoelectronic devices, with particular interests in III-V on Si wafer bonding technology, compliant substrate theory and technology, and optoelectronic devices (e.g., long wavelength VCSELs) using the above material and processing technologies. He joined the Department of Electrical and Computer Engineering, University of California, San Diego (UCSD), as a Professor in 1999. His research interests at UCSD have been in biophotonics, fluidic-photonic integrated circuits, and fluidic tunable optics, all of which can find applications in sensors and biomedicine. His group at UCSD is also involved in single-photon detector research for quantum information technology, communication, and sensing. He was a co-founder of Nova Crystals, an optoelectronic component company for telecommunication, and has served as the CTO and was on the Board of Directors until March 2004, when the company was acquired by Gemfire and its holding company. He also founded Cloverhurst Technology, in 2004, to commercialize the technology of fluidic tunable optics for miniaturized imaging systems. He has published more than 250 refereed papers in technical journals and conferences and has given many invited talks and tutorials. He was awarded with 18 patents.

Dr. Lo is currently Chairman of the Integrated Optics and Optoelectronics Committee for LEOS. He is an Associate Editor for the IEEE PHOTONICS TECHNOLOGY LETTERS, and a committee member of several international conferences.

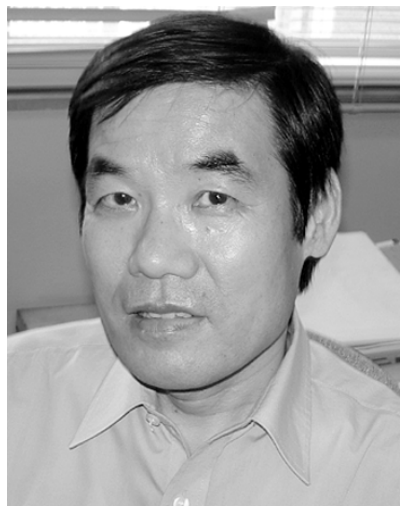

Kunishige Oe (M-87) was born in Kyoto, Japan, in 1948. He received the B.S., M.S., and Ph.D. degrees from Kyoto University, Kyoto, in 1971, 1973, and 1982, respectively.

In 1973, he joined NTT Electrical Communication Laboratories, Tokyo, Japan, where he worked on LPE growth and laser fabrication of GalnAsP/InP double-heterostructures, which resulted in the first oscillation of $1.3 \mu \mathrm{m} \mathrm{CW}$ laser diodes in 1977. He moved to electronic device research in 1983, in which he researched fabrication and characterization of heterostructures with two-dimensional gases and developed heterostructure FETs. Since 1986, he has been working on advanced semiconductor lasers and semiconductor photonic integrated circuits. From 1990 to 1991, he was a Visiting Scientist with Bell Communication Research, Red Bank, NJ. In 1998, he left NTT and became a Professor with Kyoto Institute of Technology, Kyoto. His current research interests include novel semiconductor lasers whose wavelength stays nearly constant through ambient temperature variation, photonic integrated circuits, and WDM components.

Dr. Oe is a member of the Japan Society of Applied Physics and the Institute of Electronics, Information and Communication Engineers of Japan. 


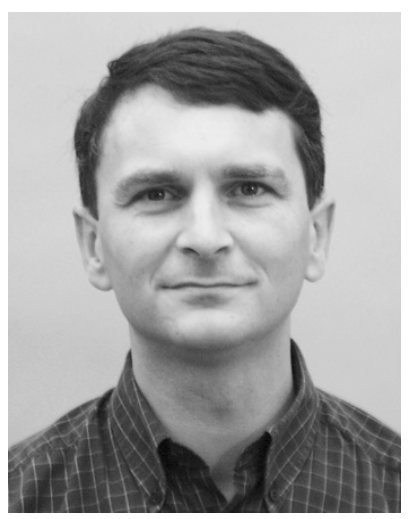

Roel Baets was born in Wommelgem, Antwerp, Belgium in 1957. He received the degree in electrical engineering, from Ghent University, Ghent, Belgium, in 1980, the M.Sc. degree in electrical engineering from Stanford University, Stanford, CA, in 1981, and the Ph.D. and the "Aggregaat Hoger Onderwijs" degrees from Ghent University, in 1984 and 1988, respectively.

Since 1981, he has been with the Department of Information Technology (INTEC, formerly Laboratory of Electromagnetism and Acoustics), Ghent University, first as doctoral student (FWO-grant) and later as an IMEC Group Leader of optoelectronic device research. Since 1989, he has been a Full-Time Professor in the Engineering Faculty, Ghent University. From 1990 to 1994, he was a Part-Time Professor with the Technical University of Delft, Delft, The Netherlands. He has mainly worked in the field of photonic components. With about 400 publications and conference papers as well as about ten patents, he has made contributions to research on III-V semiconductor laser diodes, passive guided wave and grating devices, and to the design and fabrication of photonic ICs. He leads the Photonics Research Group, Ghent University-INTEC (associated lab of IMEC), which focuses on new concepts for optoelectronic components for optical communication, optical interconnect, and optical sensing. This includes the design, fabrication, and characterization of new devices fabricated in the clean room facility at Ghent University or in collaboration with third parties. He has been involved in a number of European research projects and has been the Coordinator of several of them. He currently coordinates the European Network of Excellence ePIXnet on photonic integrated components and circuits. At Ghent University, he is teaching courses relating to optoelectronics, photonics, and optical fiber communication for electrical engineering and applied physics engineering students. He has been chairing the electrical engineering educational committee of the Faculty of Applied Sciences (1996-2000) and currently chairs the Faculty's Research Committee. He has served as a member of the program committee of the ESSDERC conference, of the IEEE International Semiconductor Laser Conference, of the European Conference on Integrated Optics, of the European Conference on Optical Communication, of the Optical Fiber Conference, of CLEO-Europe, and of IPR.

Dr. Baets is a member of IEEE-LEOS, the Optical Society of America, the International Society for Optical Engineers, and the Flemish Engineers Association. From 1999 to 2001, he was Chairman of the IEEE LEOS-Benelux chapter. Currently he is a member of the Board of Governors of IEEE LEOS. 